\title{
Low-Cost Wearable Data Acquisition for Stroke Rehabilitation: A Proof- of-Concept Study on Accelerometry for Functional Task Assessment
}

\author{
Antonio J. Salazar, MSc, ${ }^{1,2}$ Ana S. Silva, MSc, ${ }^{1,2}$ Claudia Silva, MSc, ${ }^{3}$ Carla M. Borges, Eng, ${ }^{2}$ \\ Miguel V. Correia, PhD, ${ }^{1,2,4}$ Rubim S. Santos, $\mathrm{PhD},{ }^{3}$ and Joao P. Vilas-Boas, $\mathrm{PhD}{ }^{4}$ \\ ${ }^{1}$ INESC Technology and Science (INESC TEC), Porto, Portugal; ${ }^{2}$ Faculdade de Engenharia, Universidade do Porto, Porto, Portugal; \\ ${ }^{3}$ Centro de Estudos do Movimento e Actividade Humana (CEMAH), ESTSP-IPP, Vila Nova de Gaia, Portugal; \\ ${ }^{4}$ Biomechanics Laboratory (LABIOMEP), Faculdade de Desporto, Universidade do Porto, Porto, Portugal
}

\begin{abstract}
Background: An increasingly aging society and consequently rising number of patients with poststroke-related neurological dysfunctions are forcing the rehabilitation field to adapt to ever-growing demands. Although clinical reasoning within rehabilitation is dependent on patient movement performance analysis, current strategies for monitoring rehabilitation progress are based on subjective time-consuming assessment scales, not often applied. Therefore, a need exists for efficient nonsubjective monitoring methods. Wearable monitoring devices are rapidly becoming a recognized option in rehabilitation for quantitative measures. Developments in sensors, embedded technology, and smart textile are driving rehabilitation to adopt an objective, seamless, efficient, and cost-effective delivery system. This study aims to assist physiotherapists' clinical reasoning process through the incorporation of accelerometers as part of an electronic data acquisition system. Methods: A simple, low-cost, wearable device for poststroke rehabilitation progress monitoring was developed based on commercially available inertial sensors. Accelerometry data acquisition was performed for 4 first-time poststroke patients during a reach-press-return task. Results: Preliminary studies revealed acceleration profiles of stroke patients through which it is possible to quantitatively assess the functional movement, identify compensatory strategies, and help define proper movement. Conclusion: An inertial data acquisition system was designed and developed as a low-cost option for monitoring rehabilitation. The device seeks to ease the data-gathering process by physiotherapists to complement current practices with accelerometry profiles and aid the development of quantifiable methodologies and protocols. Key words: accelerometry data, quantitative strategies, rehabilitation monitoring, stroke, wearable technology
\end{abstract}

A $\mathrm{n}$ in-depth understanding of the human body in everyday scenarios, advanced skills in physical assessment, and experience in hands-on management allow physiotherapists to manage a broad range of conditions, with the fundamental goal of promoting wellness, mobility, and independent function. ${ }^{1}$ Despite these well-stated skills, most current evaluation procedures still lack validity, reproducibility, and sensitivity because they rely mostly on clinical assessment scales, which are influenced by subjectivity. The development and implementation of objective measures within rehabilitation evaluation procedures may help enhance clinical reasoning and potentiate rehabilitation efficiency.
Until recently, most research involving the capture and analysis of biometric and/or physiological signals had been limited to a laboratory or an otherwise controlled environment, because the use of complex and costly equipment required specialized spaces and personnel. The emerging trend of wearable monitoring systems provides an opportunity to complement qualitative scales with quantitative data that are objective, digitally stored, automatically processed, and shared among the rehabilitation team in-house or remotely.

The wearable system used in the present study follows a modular approach in which selected 
sensor modules can be interconnected and used in combination to extract meaningful information according to the target application requirements.

\section{Current Rehabilitation Protocols and Procedures}

Modern technological developments for rehabilitation should consider low-cost adaptable solutions that can be integrated in everyday scenarios as opposed to rigid strategies meant for limited usability. Considering that there were an estimated 10.3 million first-ever stroke survivors worldwide in $2005^{2}$ and stroke is projected to remain a leading cause of disabilityadjusted life years ${ }^{3}$ through 2030, stroke care represents a major burden on global health care expenditures, constituting about 3\% of health care costs. ${ }^{4}$ Despite the elevated related cost, there is a general agreement on the importance of addressing the sequelae of stroke. Although there are well-established beneficial effects of timely and continuous managed rehabilitation, ${ }^{1}$ there is a need to improve the objective documentation of physical recovery, patient-tailored training programs, and the effectiveness of the rendered physiotherapy.

A crucial aspect guiding a physiotherapist's clinical reasoning, and thus design of rehabilitation intervention, is the assessment of motor performance. According to some authors, ${ }^{5}$ implementing therapeutic programs requires accurate clinical and field measurements based on motor pattern identification not readily available from traditional tools. Standardized clinical motor assessments rely on physiotherapists' observational skills, which, although valuable, remain insufficient for reliable measurement of certain quantitative features (eg, intersegment coordination, quality and smoothness of movement). Moreover, observation-based assessment is subject to observer error and personal bias and is limited to the human visual perception sensitivity. The Rivermead Motor Assessment (RMA), Fugl-Meyer Motor Assessment (FMA), Postural Assessment Scale for Stroke Patient, and Reach Performance Scale (RPS) are examples of viable and reliable assessment tools commonly used in physiotherapy. In contrast, evaluation instrumentation typically found in laboratories, including electromyography, force platforms, and image/video analysis systems, introduces a degree of objectivity in data collection, processing, and interpretation, augmenting the rehabilitation specialist's ability to characterize the patient's motor control and functional deficits. ${ }^{6-8}$ However, such resources are not available in most rehabilitation clinics or in the patients' home environment, thus depriving most patients of effective physical intervention.

Quantitative data records provide a means for efficient and expedient analysis of the effectiveness of a therapy on a patient's progress, which acts as a safeguard from negative activities that can go unnoted and unrecorded. Such an approach strengthens and streamlines internal technological platforms, expanding their coverage and added value and promoting the formulation of standards and protocols for monitoring patient progress, thus augmenting current guidelines.

\section{Inertial Monitoring System}

At the request of physiotherapists from the Escola Superior de Tecnologia de Saude do Porto in Porto, Portugal, who were pursuing alternative therapies for home-based rehabilitation for stroke survivors, a simple modular inertial monitoring device was designed and implemented based on commercially available components and sensors, such as the ADXL345 accelerometer, ITG3200 gyroscope, and the HMC5843 magnetometer. The system as shown in Figure $\mathbf{l}$ is based on a modular approach centered on the Arduino FIO, XBEE wireless module and ADXL345 accelerometer and is in an almost out-of-the-box ready-to-use condition. Swift calibration (when compared with marker-based video/image analysis equipment) through sensor positioning verification offers a reduced set-up period. Although sensor error varies depending on the particular component (eg, the ADXL345 has a sensitivity deviation from ideal and temperature of $\pm 1 \%$ and $\pm 0.01 \%$, respectively), built-in features of modern sensors (such as temperature sensors and self-testing mechanism) contribute to the reliability of the measurement. The device used for the study was validated against the Qualisys system present at the Centro de Estudos do Movimento e Actividade 

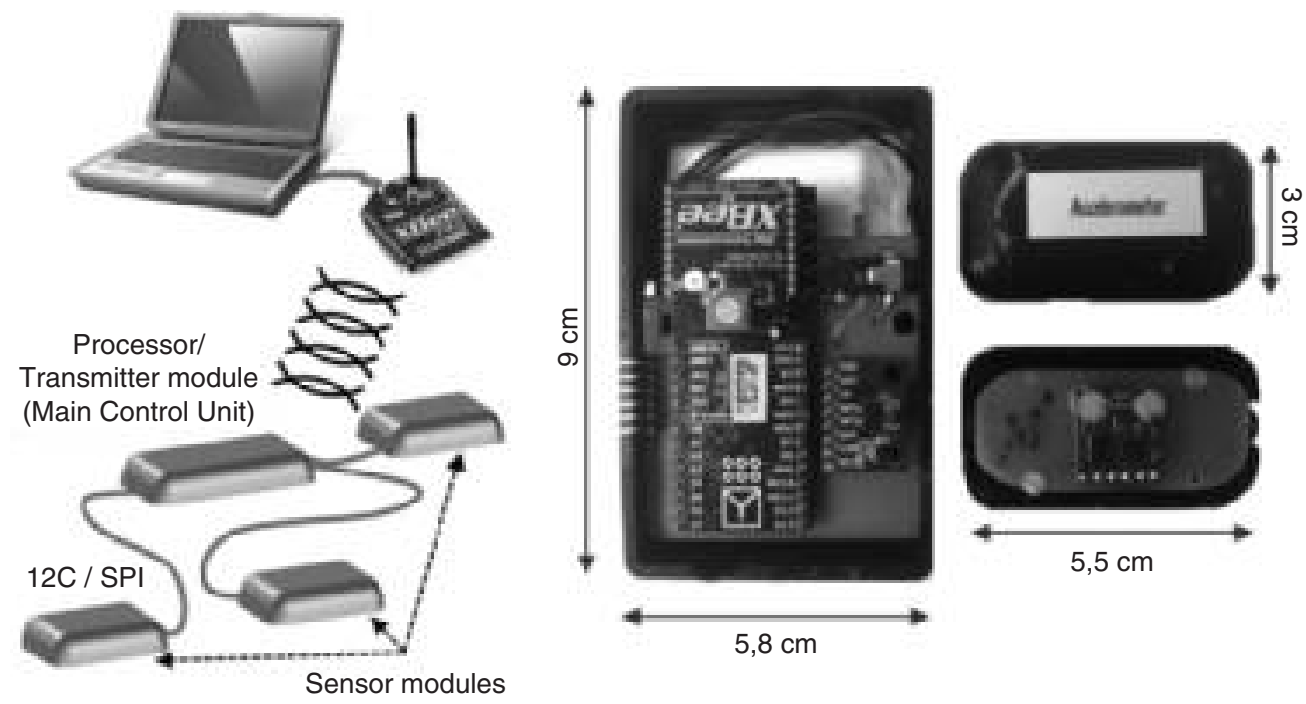

Figure 1. Data acquisition system: (left) modular approach schematic, (right) modules image (main control unit and accelerometers).

Humana facilities in collaboration with the Escola Superior de Tecnologias da Saude of Porto.

A custom processing (an open-source programming language) application provided a graphical user interface (GUI) for recording patients' information and session data; however, a number of alternative programming environments can be used for constructing such a GUI, such as LabVIEW, Python, and Visual Studios. When compared with other physiological and biological signal-monitoring devices available in the market, the solution presented can be considered low cost as it can be constructed for under $\$ 300$. Devices such as Biopac, Plux, Biodex, and videobased solutions from Qualisys and Viacon are some examples of systems being used today in rehabilitation and medical research. The use of inertial sensors for motion analysis has been gaining momentum in recent years, ${ }^{9,10}$ and some commercially available solutions now exist, such as those from Xsense and Delsys. These systems retail from thousands to hundreds of thousands of euros and tend to require equally expensive addons and training.

The ultimate goal is the implementation of a home-based rehabilitation monitoring platform as illustrated in Figure 2. The platform would not impose a radical shift from clinical management but would seek a redefinition of the physician- patient dynamics, thus promoting the generation of databases that can be shared and analyzed for an increased understanding of people's responses to their surroundings under a number of scenarios.

\section{Experimental Protocol}

Four stroke survivors participated in a proofof-concept study. The patients were informed of the experimental procedures and provided written consent in accordance with policies of the institution's ethics committee. Participants had to meet the following inclusion criteria:

- Confirmatory neuroimaging results of a single, unilateral stroke in the medial cerebral artery (MCA) territory, sustained at least 3 months prior

- Absence of hemispatial neglect

- Absence of major visual, perceptual, or cognitive deficits, confirmed by a MiniMental State Examination score of 24

- Active range of motion in the compromised arm of at least 15 in the shoulder (flexion/ extension, abduction/adduction, and internal/ external rotation) and elbow (flexion/ extension $)^{11}$

Explicit exclusion criteria included cerebellar or brain stem lesions and pain/subluxation of the shoulder. Arm motor impairment was evaluated prior 


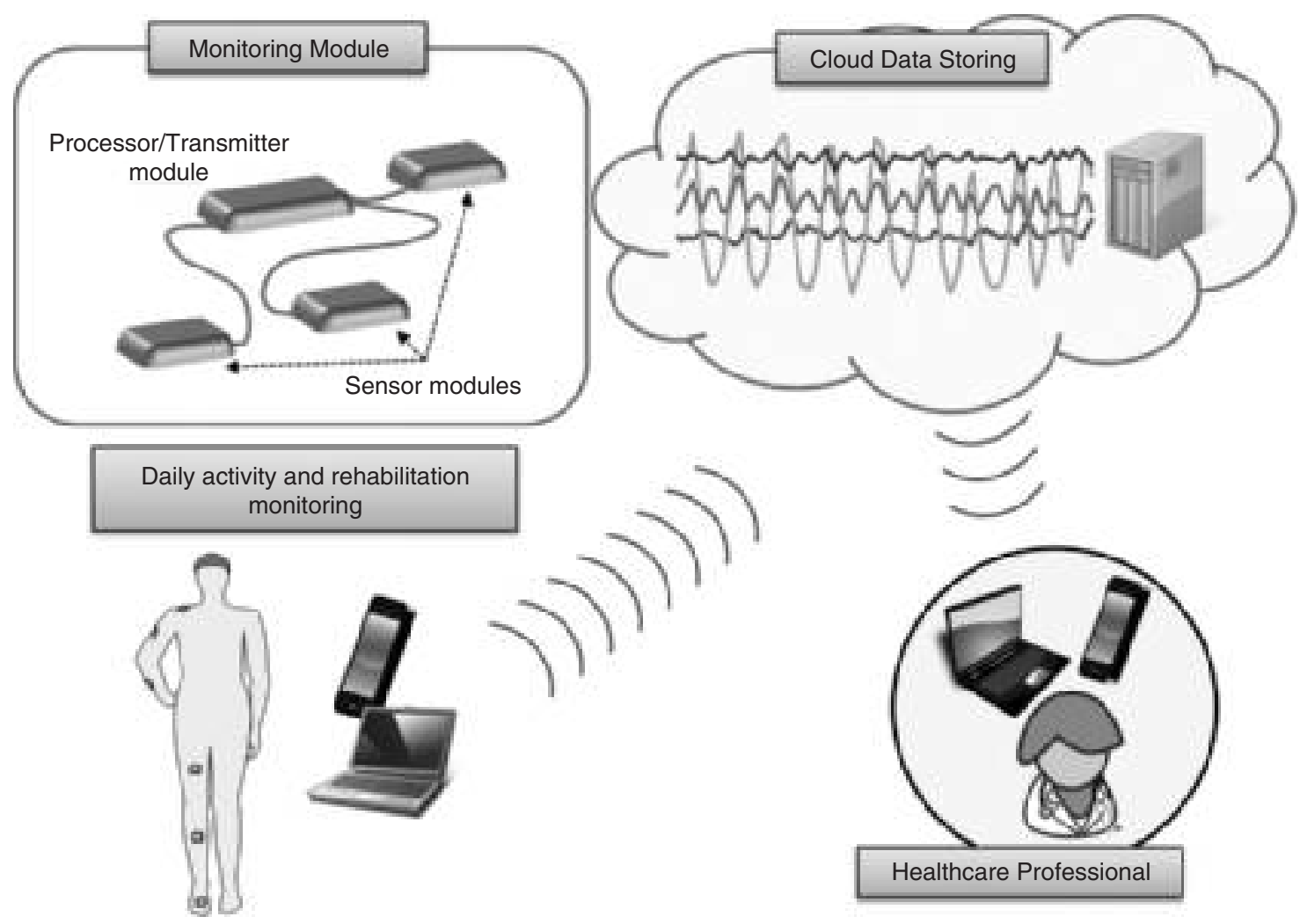

Figure 2. Data acquisition platform illustration.

to measurements, as seen in Table $\mathbf{1}$, with the arm subsection of the FMA ${ }^{12}$ and the RPS ${ }^{13}$ (close target).

Each subject was assessed in sitting position. A table was placed in front of the subject at a height corresponding to the alignment of the iliac crests. The proximal table limit was coincident with the distal border of the subject's knees, so as not to interfere with the arm trajectory. The subjects started the task with approximately $0^{\circ}$ of flexion/extension/internal rotation at the shoulder, approximately $100^{\circ}$ of flexion at the elbow, with the forearm in pronation and the palm of the hand resting on the thigh. The subjects were instructed to reach and press a target placed ipsilaterally to the upper limb in the study, in groups of 3 repetitions (to avoid variations caused by fatigue) separated by 1-minute rest periods. The target's placement reference was the anatomical reaching distance of the hand, with the measured distance from the acromion to the metacarpophalangeal joint of the thumb. ${ }^{14}$ The subjects were instructed, after verbal command, to perform reaching.
Table 1. Demographic data and clinical scores of stroke patients

\begin{tabular}{lllll}
\hline & \multicolumn{4}{c}{ Subjects } \\
\cline { 2 - 5 } & A & B & C & D \\
\hline Age in years/gender & 64/M & $47 / \mathrm{F}$ & $53 / \mathrm{F}$ & $49 / \mathrm{M}$ \\
Location of lesion & LMCA & RMCA & LMCA & LMCA \\
Months post stroke & 19 & 20 & 34 & 66 \\
RPS score & $7 / 18$ & $12 / 18$ & $8 / 18$ & $5 / 18$ \\
FMA (shoulder, elbow, & $8 / 36$ & $20 / 36$ & $17 / 36$ & $4 / 36$ \\
forearm) & & & & \\
FMA (wrist) & $1 / 10$ & $2 / 10$ & $2 / 10$ & $0 / 10$ \\
FMA (hand) & $6 / 14$ & $12 / 14$ & $10 / 14$ & $2 / 14$ \\
FMA (coordination) & $1 / 6$ & $3 / 6$ & $4 / 6$ & $0 / 6$ \\
& & & & \\
\hline
\end{tabular}

Note: FMA = Fugl-Meyer Assessment; LMCA = left medial cerebral artery; RMCA = right medial cerebral artery; RPS = Reach Performance Scale (close target).

Precise bone landmarks were required to ensure sensor placement repeatability. A complementary study $^{15}$ that focused on determining appropriate sensor positions for the detection of compensatory movements (illustrated in Figure 3) suggested 

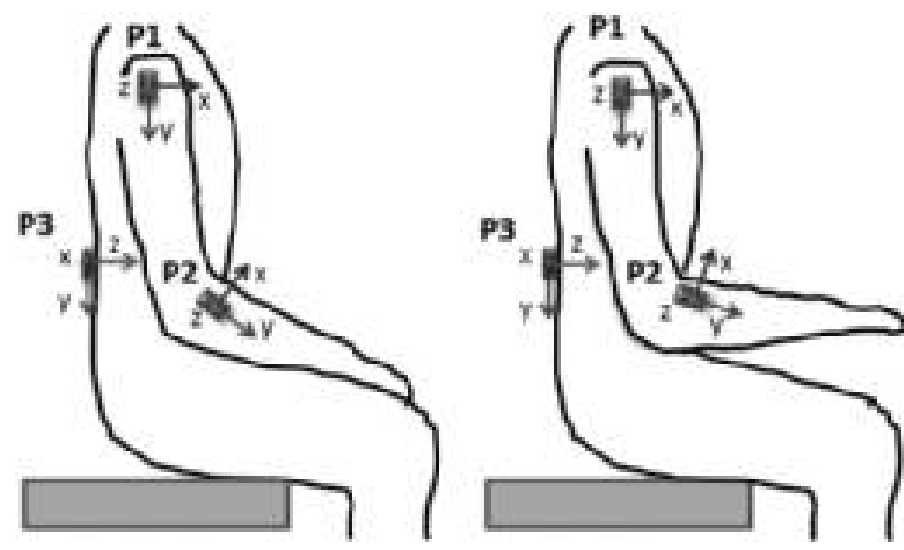

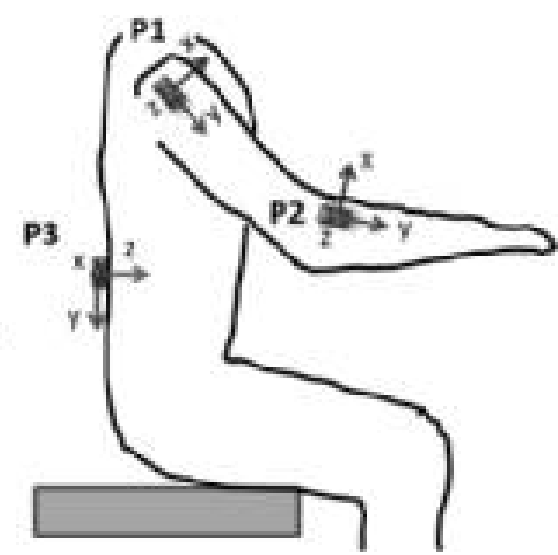

Figure 3. Reach phases and sensor locations.

that the position referred to as $\mathrm{Pl}$ is advantageous for compensatory elevation movement detection at the shoulder; P2 is suitable for detecting the abduction; and P3 presents a reasonable sensitivity for detection of forward dislocation and rotation of the trunk. Following these results, the positions chosen for the present study are as follows:

- P1, placed under the acromion, following the line that connects the lateral epicondyle and the acromion

- P2, immediately below the lateral epicondyle, after elbow articulation

- P3, on the trunk at the T12 vertebrae

\section{Accelerometry analysis}

As mentioned earlier, human movement accelerometry analysis has gained momentum, including in its applicability for stroke rehabilitation, ${ }^{16,17}$ aided by the commercialization of microelectromechanical system (MEMS) accelerometers. In general, MEMS accelerometers measure the deviation from free fall; that is, their output represents the vector sum of the gravitational and kinematic accelerations of selfmovement. Rehabilitation scenarios generally present small sporadic dynamic acceleration, related to the wearer's movements, when compared with gravitational acceleration components. A quasi-static model can be adopted when the dynamic acceleration contribution is negligible; the application of such assumption has revealed reasonable results when compared with videobased systems. ${ }^{18,19}$
The accelerometry data for the present study were acquired at a $100 \mathrm{~Hz}$ sampling frequency, packed (data from all 3-axis accelerometers), time-stamped, and transmitted wirelessly to a remote station. A 50-point symmetrical moving average filter was then applied for dynamic acceleration and artifact removal, and movement start/end markers were automatically determined by the selection of $\mathrm{min} / \mathrm{max}$ points generated by the application of a symmetric 2-point numerical differentiation on all axes, such that

$S^{\prime}(t) \geq \alpha * \mid \max \left(S^{\prime}(t)\right)-$ mean $\left(S^{\prime}(t)\right) \mid+$ mean $\left(S^{\prime}(t)\right)$.

Experimentation revealed that a span of $300 \mathrm{~ms}$ and $\alpha=0.3$ offered consistent results in determining the start/end of the movement. Additional plus/ minus pseudo-envelope functions were generated by using a moving window standard deviation approach (with a window of $0.3 \mathrm{~s}$ ), which served as visual indicators of signal deviation strength. Finally, the data were time-normalized for intraand interpatient comparability.

The generated accelerometry profile provides a visual representation of the completed functional task, which can be associated with angular displacement through spherical conversion following Equations 1 and 2. Such equations relate to the angle of tilt of the transverse plane and the inclination of the longitudinal axis from the gravity vector, respectively (considering a reference frame coinciding with the anterior-posterior axis, the mediolateral axis, and the longitudinal axis of the cardinal planes as the $\mathrm{x}^{-}, \mathrm{y}-$, and $\mathrm{z}$-axis, 
respectively). The inherent relation of the data with angular displacement serves as a basis for event/data correlation.

$$
\begin{aligned}
& \theta=\tan ^{-1}\left(\frac{A c c_{x}}{A c c_{y}}\right) \\
& \phi=\cos ^{-1}\left(\frac{A c c_{z}}{\sqrt{A c c_{x}^{2}+A c c_{y}^{2}+A c c_{z}^{2}}}\right)
\end{aligned}
$$

It should be mentioned that a number of strategies are being applied to inertial sensors for human movement analysis; however, the present study focused on the aforementioned accelerometry profiles as a source for the extraction of movement performance quantifiers, because they were easy to obtain and were able to be associated with physical events by direct inspection.

An example of an accelerometry profile of the processed data, seen in Figures $4 \mathrm{~A}$ and $\mathrm{B}$ in a real-number three-dimensional representation, illustrates the difference between 3 independent reach-press-return repetitions performed by a reference subject with no neural or musculoskeletal pathologies (namely at cervical spine and upperlimb levels) and a poststroke subject, respectively.
Clear differences are observed in Figures 4A and $\mathbf{B}$ between the movement path of the reference subject and the poststroke subject, namely the path smoothness and the consistency of the performed task. In fact, the jerkiness and the variability among the repetitions observed in the poststroke individual data, in both reach and return phases and also among different trials, may reflect motor control deficits and biomechanical constraints resulting from central nervous system injury. Brain areas supplied by MCA, both cortical and subcortical, may present lesion and/ or dysfunction after a stroke, interfering with the adequate sequence of events known to occur in typical reaching.

For a more exhaustive analysis, data were collected from neurologically intact subjects for the reach-press-return functional task and confirmed visually by a physiotherapist for "proper" movement performance. A set of extracted reference signals from the accelerometers positioned at positions $\mathrm{P} 1, \mathrm{P} 2$, and $\mathrm{P} 3$ are presented in Figure 5. Similarly, accelerometry data were recorded from poststroke patients referred to as A (Figure 6), B (Figure 7), C (Figure 8), and D (Figure 9) for all mentioned positions. In Figures 5-9, the solid lines represent the processed signal, the black dashed lines represent the plus/minus envelope signals, and the gray
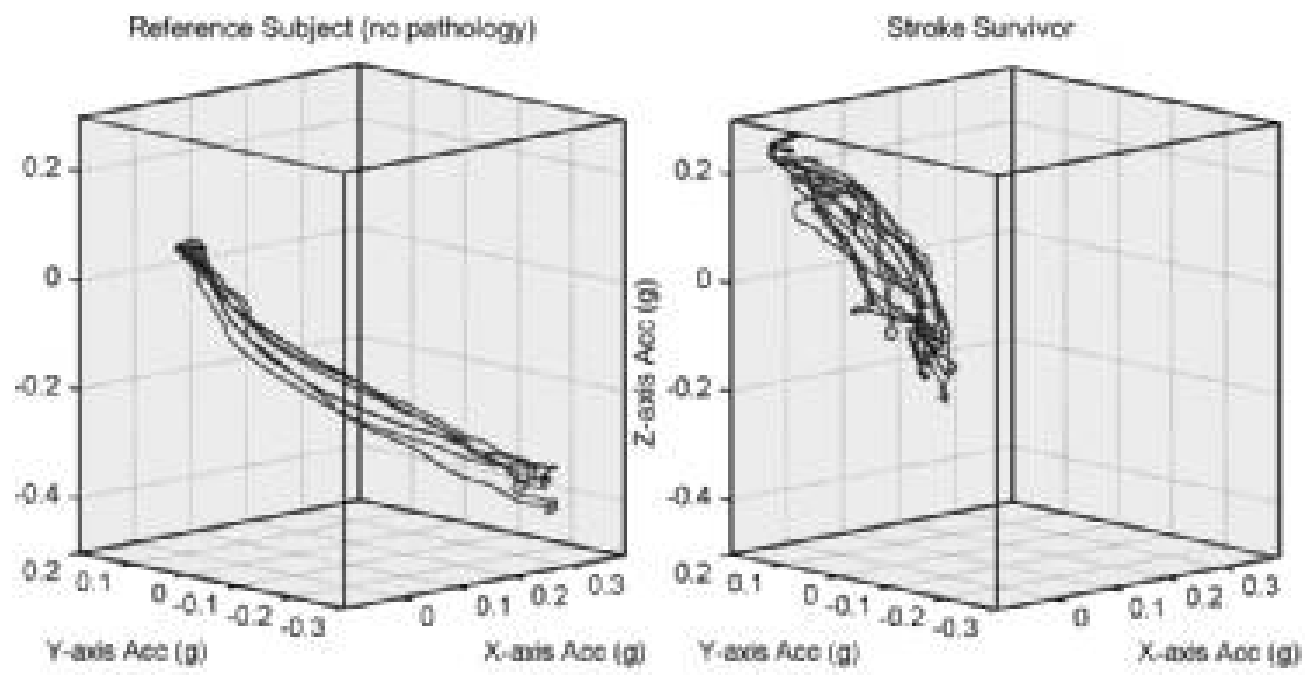

Figure 4. Accelerometry (Acc) profile of 3 repetitions for reach-press-return for (a) reference subject and (b) stroke survivor. 

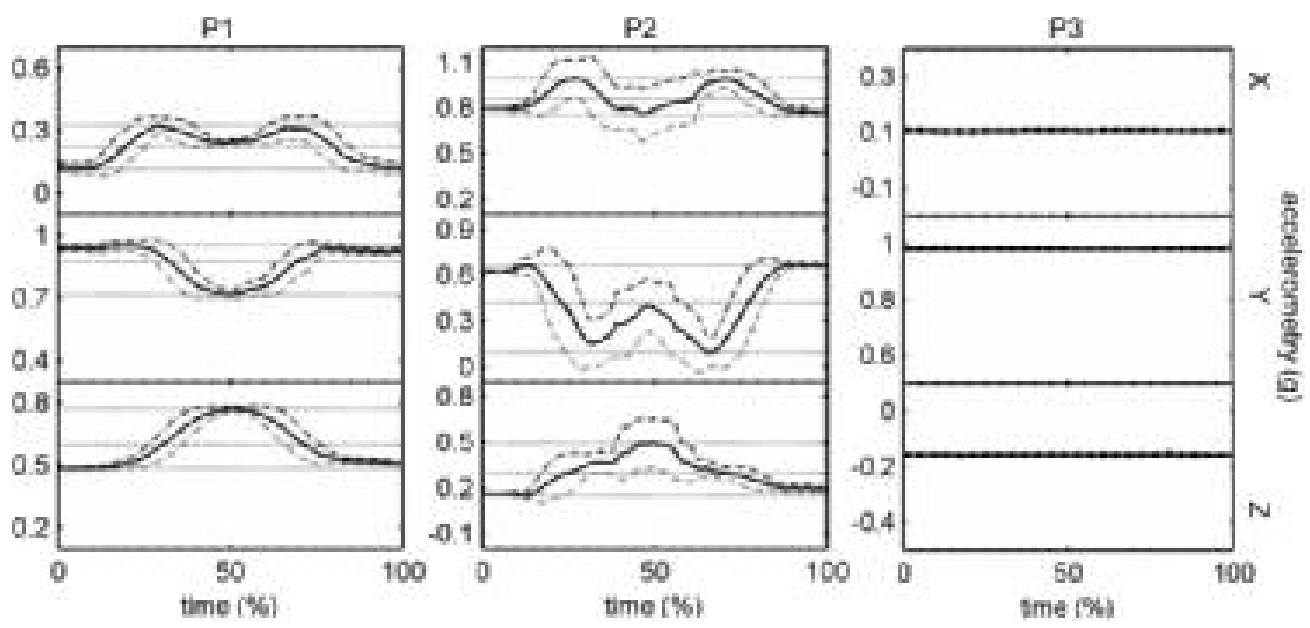

Figure 5. Accelerometry data for subject without pathology in positions P1, P2, and P3.
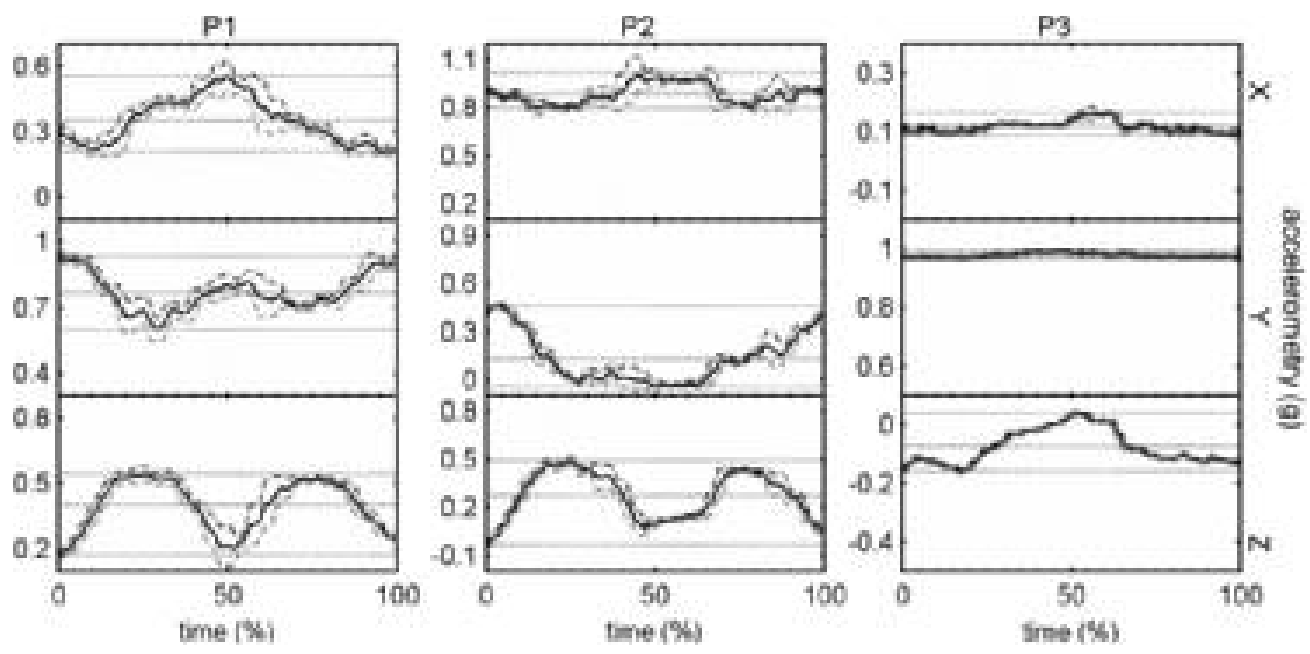

Figure 6. Accelerometry data for subject $\mathrm{A}$ in positions $\mathrm{P} 1, \mathrm{P} 2$, and $\mathrm{P} 3$.
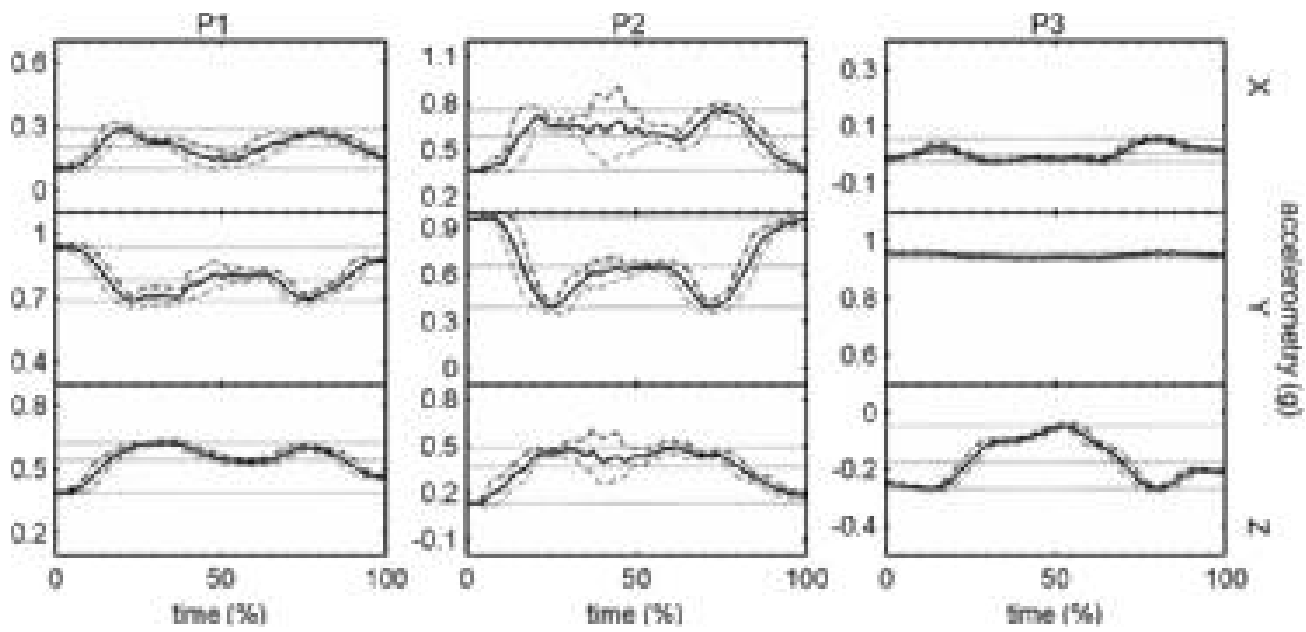

Figure 7. Accelerometry data for subject B in positions P1, P2, and P3. 

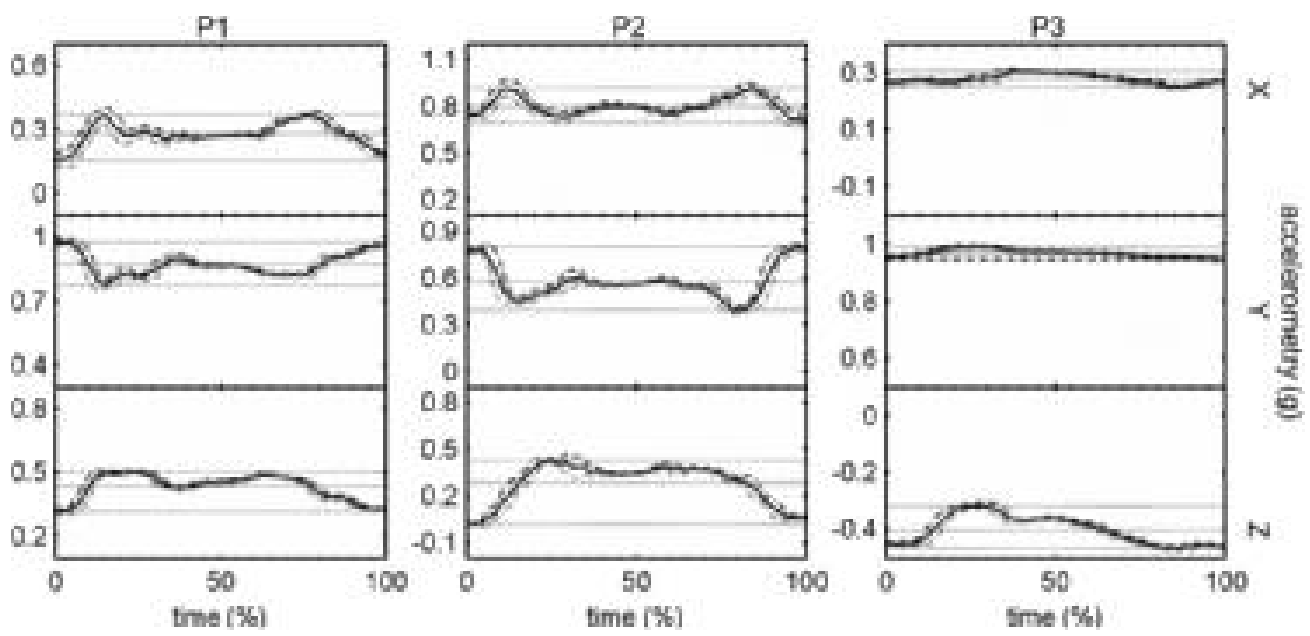

Figure 8. Accelerometry data for subject $\mathrm{C}$ in positions P1, P2, and P3.
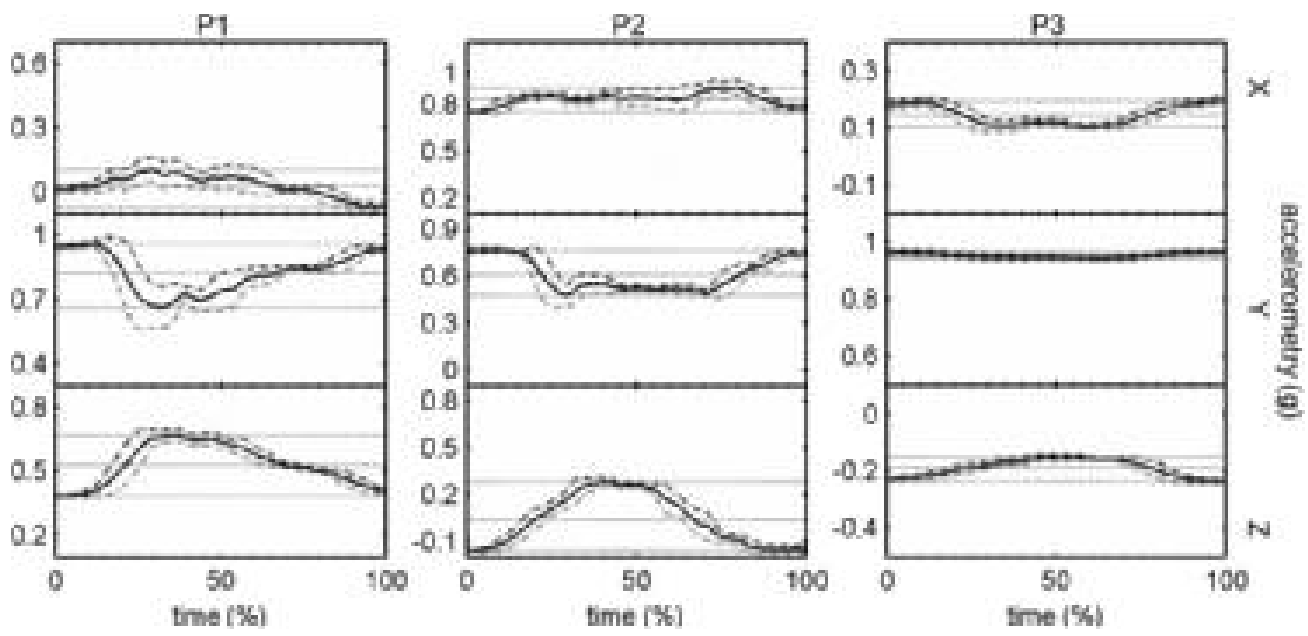

Figure 9. Accelerometry data for subject D in positions P1, P2, and P3.

dashed lines represent the maximum, minimum, and mean values of the processed signal. Each position has its corresponding acceleration graphs (measured in gs) presenting information for all 3 axes. In general, the subjects present a proximal to distal sequence of muscular requirement, varying degrees of deficit of elbow extension, and trunk recruitment for task completion.

Table 2 summarizes the observations extracted from the data collected, direct observation, and corresponding video records. For instance, trunk compensation, either through forward dislocation or rotation, can be well detected by data collected from accelerometers at position P3. At this location, the reference subject's accelerometry data reveal small variations during the entire movement, caused by small shifts in the center of mass. In contrast, stroke patients show a perceived dislocation of the trunk: an upper movement to assist with the forearm elevation and a forward dislocation and rotation to compensate for lack of full elbow extension.

In general, the differences observed in the accelerometry profiles can be related to the presence of compensatory movements. For instance, stroke survivor subjects' y-axis profiles at position $\mathrm{Pl}$ (which are clearly dissimilar from the corresponding reference profile because of the presence of a double bell shape for subjects $\mathrm{A}, \mathrm{B}$, and $\mathrm{C}$ and an asymmetrical bell shape for 
Table 2. Summary of accelerometry profiles observations

\begin{tabular}{lllll}
\hline & x-axis & y-axis & z-axis & $\begin{array}{l}\text { Movement } \\
\text { compensations }\end{array}$ \\
\hline P1 & $\begin{array}{l}\text { Shoulder angular } \\
\text { displacement on the } \\
\text { anterior/posterior direction }\end{array}$ & $\begin{array}{l}\text { Shoulder angular } \\
\text { displacement on the } \\
\text { superior/inferior direction }\end{array}$ & $\begin{array}{l}\text { Shoulder angular } \\
\text { displacement on the } \\
\text { medial/lateral direction }\end{array}$ & $\begin{array}{l}\text { Shoulder } \\
\text { abduction and } \\
\text { elevation }\end{array}$ \\
P2 & $\begin{array}{l}\text { Forearm angular } \\
\text { displacement information } \\
\text { on the superior/inferior } \\
\text { direction }\end{array}$ & $\begin{array}{l}\text { Forearm angular } \\
\text { displacement information } \\
\text { on the anterior/posterior } \\
\text { direction }\end{array}$ & $\begin{array}{l}\text { Forearm angular } \\
\text { displacement } \\
\text { information lateral } \\
\text { movement }\end{array}$ & $\begin{array}{l}\text { Lack of elbow } \\
\text { extension } \\
\text { and shoulder } \\
\text { abduction }\end{array}$ \\
& $\begin{array}{l}\text { Trunk angular } \\
\text { displacement on the } \\
\text { superior/inferior direction }\end{array}$ & $\begin{array}{l}\text { Trunk angular } \\
\text { displacement on the } \\
\text { anterior/posterior direction }\end{array}$ & $\begin{array}{l}\text { Forward } \\
\text { dislocation and } \\
\text { trunk rotation }\end{array}$ \\
\end{tabular}

${ }^{\mathrm{a}}$ Needs confirmation with additional information from P3.

Table 3. Extracted quantifiers from accelerometry profiles

\begin{tabular}{llrrrrr}
\hline & & \multicolumn{5}{c}{ Subjects } \\
\cline { 3 - 7 } Postition & & \multicolumn{1}{c}{ A } & \multicolumn{1}{c}{ B } & \multicolumn{1}{c}{ C } & D & Ref \\
\hline \multirow{2}{*}{ P1 } & Avg. $\Delta$ x-axis $(g)$ & 0.39 & 0.17 & 0.21 & 0.14 & 0.21 \\
& Avg. $\Delta$ y-axis $(g)$ & 0.42 & 0.25 & 0.20 & 0.26 & 0.22 \\
& Avg. $\Delta$ z-axis $(g)$ & 0.42 & 0.24 & 0.20 & 0.25 & 0.28 \\
& Mean comparability factor & 0.14 & 0.27 & 0.26 & 0.72 & $0.98^{\text {a }}$ \\
& Repeatability factor & 13.89 & 3.63 & 6.54 & 3.83 & 2.47 \\
\hline \multirow{2}{*}{ P2 } & Avg. $\Delta$ x-axis $(g)$ & 0.23 & 0.45 & 0.32 & 0.17 & 0.23 \\
& Avg. $\Delta$ y-axis $(g)$ & 0.50 & 0.62 & 0.35 & 0.31 & 0.57 \\
& Avg. $\Delta$ z-axis $(g)$ & 0.53 & 0.37 & 0.45 & 0.45 & 0.33 \\
& Mean comparability factor & 0.47 & 0.60 & 0.34 & 0.69 & $0.98^{\text {a }}$ \\
& Repeatability factor & 5.85 & 10.65 & 11.36 & 6.34 & 6.34 \\
\hline \multirow{2}{*}{ P3 } & Avg. $\Delta$ x-axis $(g)$ & 0.09 & 0.08 & 0.11 & 0.09 & 0.04 \\
& Avg. $\Delta$ y-axis $(g)$ & 0.03 & 0.02 & 0.17 & 0.02 & 0.01 \\
& Avg. $\Delta$ z-axis $(g)$ & 0.23 & 0.22 & 0.23 & 0.09 & 0.01 \\
& Mean comparability factor ${ }^{b}$ & 0.99 & 0.99 & 0.96 & 0.99 & $\sim 1^{\mathrm{a}}$ \\
& Repeatability factor & 4.76 & 5.57 & 2.59 & 1.83 & 1.27 \\
\hline \multirow{2}{*}{ Average time } & (seconds) & 12.60 & 5.62 & 7.02 & 3.15 & 3.56 \\
\hline
\end{tabular}

\footnotetext{
${ }^{a}$ The calculation was performed using 3 trials from the reference individual (Ref), different from the reference signal.

${ }^{b}$ Due to the reduced variations of the signals, the offset removal was not applied in this scenario.
}

subject D) can be attributed to a shoulder and possible trunk compensatory elevation. Care must be taken when extracting quantifiers, because they could ignore specific maladaptive behaviors. For instance, subject B presented a strong tremor when approaching the press target, which can be observed by a noticeable pseudo-envelope deviation near the 40\% mark for the $\mathrm{x}$ - and $\mathrm{z}$-axes of position P2. Such tremor is indicative of a lack of proper muscular organization to complete the task and can be monitored by measuring the magnitude of the deviation.

Table 3 summarizes some quantifiers extracted from the generated accelerometry profiles, including variations on the different axes (relatable to maximum angular displacement), mean comparability factor, repeatability factor, and average execution time. The mean comparability factor was obtained, for position P1 and P2, through a standard normalized zero-lag cross- 
correlation between a reference vector and vectors generated by the grouping of centered (offset removed) accelerometry data of all axes. Because of the reduced variations of the signals for position P3, the offset removal was not performed prior to the cross-correlation calculation. The repeatability factor, presented in Table 3, represents a measure of consistency of the subject's movement components while performing the functional task. This measure was calculated based on a repeatability index applied in gait analysis, ${ }^{20}$ analogous to the American National Standards Institute procedure for obtaining a repeatability index for industrial robots. ${ }^{21}$ Equation 3 was used for the factor calculation where $n=100$ (representing the length of the data that was time normalized) and $i=3$ (representing the number of repetitions), for the present cases.

Repeatability

$$
=\frac{1}{n} \sqrt{\sum_{k}^{\{x, y, z\}}\left(\int_{0}^{n}\left[\max _{\forall i} F_{k}^{i}(l)-\min _{\forall i} F_{k}^{i}(l)\right] d l\right)^{2}}
$$

where $F_{k}^{i}$ is the $k$ component of the ith repetition and $k \in\{\mathrm{x}, \mathrm{y}, \mathrm{z}\}$.

Overall analysis of the acceleration variations shows that for certain scenarios, poststroke subjects recruit additional degrees of freedom for task completion when compared with the reference signals. For instance, the y-axis delta values at $\mathrm{Pl}$ and $z$-axis delta values at P2 are consistent with observed excessive elevation and abduction of the shoulder performed by the patients. The mean comparability and repeatability factors for $\mathrm{Pl}$ are consistent with the FMA and RPS overall scores; however, positions $\mathrm{P} 2$ and $\mathrm{P} 3$ are more sensitive to the specific recruited strategies of each individual and permit some insight on the performance of the elbow and trunk regions, respectively.

As with most indicators, the presented quantifiers should be analyzed in context, because the data obtained from a subject could be misconstrued as being associated with a process of motor refinement when, in reality, it may represent a maladaptive behavior. A particular case occurred for subject D. Although his FMA and RPS scores showed that subject D was the most impaired patient, he presented high comparability and consistency compared with the rest of the group. A possible explanation is the extension of his poststroke period (the patient experienced his stroke almost 6 years before study) and his severe biomechanical impairments at the arm's joints and at soft tissues levels, which compromised his ability to successfully accomplish the task. The anticipation of task failure had accustomed the patient to avoid recruiting additional degrees of freedom (observed on the delta information for positions $\mathrm{P} 1$ and P2), thus forcing him to incorporate a maladaptive behavior to his reaching approach (which with time has become consistent).

It is still early to establish a simple and straightforward comparison between accelerometry profiles, because a large number of subjects, both pathological and nonpathological, would need to be assessed before proper validation is achieved. Nevertheless, the present data reveal the potential to quantitatively characterize the functional movement and establish parameters that complement observations from physiotherapists to determine compensatory behavior and proper behavioral progression.

\section{Conclusion}

An inertial data acquisition system was designed and developed as a low-cost, fast implementable option for monitoring rehabilitation progress. Although the device was originally meant for poststroke upper limb rehabilitation monitoring, its flexibility and adaptability allow its usage in a number of monitoring purposes. The device seeks to ease the data-gathering process by physiotherapists to facilitate the development of quantifiable methodologies and protocols. The presented accelerometry profiles and quantifiers can be obtained from patients while remaining under the platform's coverage even outside the clinical environment and while performing daily activities, providing the physiotherapist with useful information for the clinical reasoning process. It will be through data gathering and study of daily-life activities of a wide variety of individuals, unencumbered by the artificiality of laboratories, that fast-paced progress can be made for establishing effective and efficient rehabilitation methodologies. 


\section{REFERENCES}

1. Tate D. The state of rehabilitation research: Art or science? Arch Phys Med Rehabil. 2006;87:160-166.

2. Strong K, Mathers CD, Bonita R. Preventing stroke: Saving lives around the world. Lancet Neurol. 2007;6:182-187.

3. Lopez AD, Mathers CD, Ezzati M, Jamison DT, Murray C. Global and regional burden of disease and risk factors, 2001: Systematic analysis of population health data. Lancet. 2006;367:1747-1757.

4. Evers SMAA, Struijs JN, Ament AJHA, et al. International comparison of stroke cost studies. Stroke. 2004;35:1209-1215.

5. Patel S, Hughes R, Hester T, et al. Tracking motor recovery in stroke survivors undergoing rehabilitation using wearable technology. Conf Proc IEEE Eng Med Biol Soc. 2010;2010:6858-6861.

6. Gilmore $P$, Spaulding S. Motor learning and the use of videotape feedback after stroke. Top Stroke Rehabil. 2007;14:28-36.

7. Lucca L. Virtual reality and motor rehabilitation of the upper limb after stroke: A generation of progress? J Rehabili Med. 2009;41:1003-1006.

8. Sveistrup $\mathrm{H}$. Motor rehabilitation using virtual reality. J Neuroengin Rehabil. 2004;1:1-8.

9. Godfrey A, Conway R, Meagher D, OLaighin G. Direct measurement of human movement by accelerometry. Med Eng Phys. 2008;30(10): 1364-1386.

10. Mathie MJ, Coster AC, Lovell NH, Celler BG. Accelerometry: Providing an integrated, practical method for long-term, ambulatory monitoring of human movement. Physiol Meas. 2004;25(2): R1-R20.

11. Zachowski K, Dromerick A, Sahrmann SA, AJtion do strength, sensation, spasticity and joint individuation relate to the reaching deficits of people with chronic hemiparesis? Brain. 2004;127:7035 1046.

12. Fugl-Meyer AR, Jaasko L, Leyman I, Olsson S, Steglind S. The post-stroke hemiplegic patient: A method for evaluation of physical performance. Scand J Rehabil Med. 1975;7:13-31.
13. Levin MF, Desrosiers I, Beauchemin D, Bergeron $\mathrm{N}$, Roschette A. Development and validation of a scale for rating motor compensations used for reaching patients with hemiparesis: The Reaching Performance Scale. Phys Ther. 2004;84:8-22.

14. Vandenberghe A, Levin O, De Schutter D, Swinnen $S$, Jonkers I. Three-dimensional reaching tasks: Effect of reaching height and width on upper limb kinematics and muscle activity. Gait Posture. 2010;32:500-507.

15. Borges CM, Silva C, Salazar AJ, et al. Compensatory movement detection through inertial sensor positioning for post-stroke rehabilitation. In: Program and abstracts of the International Conference on Bio-inspired Systems and Signal Processing (BIOSIGNALS2012) February 1-4, 2010; Vilamoura, Portugal; 2010:297-302.

16. Knorr B, Hughes R, Sherrill D, Stein J, Akay M, Bonato P. Quantitative measures of functional upper limb movement in persons after stroke. In: Program and abstrational IEEE EMBS Conference on Neural Engineering; 2005: 252-255.

17. Serge SH, Cheng MS, Chang S, et al. A combined sEMG and accelerometer system for monitoring functional activity in stroke. Transact Neural Syst Rehabil Eng. 2009;17(6):585-594.

18. Bergmann JHM, Mayagoitia RE, Smith ICH. A portable system for collecting anatomical joint angles during stair ascent: A comparison with an optical tracking device. Dyn Med. 2009;8:3.

19. Pansiot J, Lo B, Guang-Zhong Y. Swimming stroke kinematic analysis with BSN. In: Proceedings of the International Conference on Body Sensor Networks (BSN); June 7-9, 2010; Singapore:153-158.

20. Bravo R, Salazar A, Basso DM, Borges C. Propuesta preliminary de un índice de consistencia para patrones de cinemática de marcha humana. Presented at the Venezuelan Bioengineering Congress 2012, BIOVEN.

21. Jeswiet J, Helferty R. Measuring robot repeatability an application of ISO and ANSI standards. Adv Robotics. 1995;10(5):503-520. 